\title{
Fast Calculation of the Light Differential Scattering Cross Section of Optically Soft and Convex Bodies
}

\author{
GRUY Frédéric \\ Ecole Nationale Supérieure des Mines, 158 Cours Fauriel, 42023 Saint-Etienne, \\ Cedex 2, France \\ gruy@emse.fr
}

\begin{abstract}
Depending on the range of size and the refractive index value, an optically soft particle follows Rayleigh-Debye-Gans or RDG approximation or Van de Hulst approximation. Practically the first one is valid for small particles whereas the second one works for large particles. Klett and Sutherland (Klett JD, Sutherland RA. Applied Optics 1992;31:373-386) proved that the Wentzel-Kramers-Brillouin or WKB approximation leads to accurate values of the differential scattering cross section of sphere and cylinder over a wide range of size. In this paper we extend the work of Klett and Sutherland by proposing a method allowing a fast calculation of the differential scattering cross section for any shape of particle with a given orientation and illuminated by an unpolarized light. Our method is based on a geometrical approximation of the particle by replacing each geometrical cross section by an ellipse and then by exactly evaluating the differential scattering cross section of the newly generated body. The latter one only contains two single integrals.
\end{abstract}

Keywords: convex body, light scattering, Rayleigh-Debye-Gans approximation, anomalous diffraction approximation, WKB approximation

\section{Introduction}

During the monitoring of particle synthesis in an industrial process, the optical properties of the suspension are measured by means of an experimental set-up composed of a monochromatic or polychromatic light source, a sampling cell and a 
photodetector array. The incident light is scattered by the sample, i.e. the suspension. Then, either the light intensity can be recorded at various scattering direction, or the transmitted intensity, i.e. the non-scattered and non-absorbed light. For suspension with identical particles, the scattered intensity is proportional to the concentration and the differential scattering cross section of the particles, whereas the transmitted light intensity is related to the particle concentration and the light extinction cross section. If the particle concentration is known, the extinction cross section is obtained through a turbidity measurement by means of the Beer-Lambert law for diluted suspension. In fact, the suspension consists of particles having different sizes and morphologies. The turbidity spectrum or the angular scattered intensity distribution of such a suspension must be analyzed during the precipitation process. The corresponding data have to be treated to deduce the concentration of each class of particle. Those are characterized by their total or differential scattering or extinction cross sections. For instance, the measured quantity is the turbidity at several wavelengths, the known parameters are the extinction cross sections and the unknown variables are the concentrations. As a consequence, the solving of the inverse problem requires previously the calculation of the optical properties of each class of particle. The exact calculation of any shapely particle is possible by numerical methods [1]. However, the whole analysis needs a too large computational time if these exact methods are used for the calculation of the optical properties. Moreover, rigorous numerical simulations are not mandatory because the particle shape, the agglomerate structure and maybe even the relevant material properties are not available with precision in such reactor. Thus simple and accurate expressions for the optical properties of particles or crystals are useful to solve inverse problems coming from such optical particle sizing techniques.

The optical properties of a body depend on the relative refractive index $m$, i.e. the ratio between the refractive indices of the particle and the surrounding medium. Some scatterers dispersed in a liquid are characterized by a low optical contrast, i.e. $|m-1|<<1$. For instance, minerals like amorphous silica, silicates, carbonates, carboxylates, ice and gas hydrate crystals in water are optically soft. Likewise, biological cells or microorganisms in water behave so. By the past, two important analytical approximations [2] have been proposed for such particles:

- Rayleigh-Debye-Gans (RDG) approximation considers small soft particles with small phase shift, namely: $2 \pi d / \lambda|m-1| \ll<1$ where $d$ and $\lambda$ are respectively the particle size and the light wavelength. Generally, the object can be divided into 
smaller identical parts (elements). Each element is polarisable. In the presence of a variable electric field, the element becomes an oscillating dipole, which itself creates an electromagnetic field. When an object is illuminated by an electromagnetic wave, each element receives the incident electric field and the one coming from the other elements. In the Rayleigh-Debye-Gans domain, the latter contribution is negligible. As a result an oscillating dipole moment may be associated to each element. Thus the object emits an electromagnetic wave (scattered wave), which includes the contribution of each oscillating dipole. In the Rayleigh-Debye-Gans domain, there is only interference of light waves that are independently scattered by all small volume elements.

- The Van de Hulst approximation, also known as anomalous diffraction (AD) approximation, because, for low optical contrast, the light passing through the particle (transmitted without deflection) interferes with that diffracted, then producing a diffraction pattern known as anomalous. Such an approximation is valid if $\pi d / \lambda>>1$ and $|m-1|<<1$. This implies that the rays are not deviated when they cross the interface particle-medium and that the reflection at this interface is negligible. Extinction is thus due to the absorption of the light passing through the particles and the interferences between the transmitted light and the incident light.

From a geometrical point of view, RDG approximation considers the distance distribution (DD) of scattering elements whereas the expression for the AD approximation contains the chord length distribution (CLD) of the total scatterer. The distance is the length between two points inside the body whereas the chord length is calculated between two points located on the body surface.

As the two approximations seem very different some investigators tried to reconcile them by an unique expression valid for $|m-1|<<1$. Perelman [3] proposed a general method and only applied it to spheres and cylinders. Klett and Sutherland [4] developed an approximate method based on the Wentzel-Kramers-Brillouin (WKB) approximation. Their approach is applied to the calculation of the phase function of spheres and cylinders [4]. In particular they mentioned that some integrations become rapidly difficult to achieve when the particle shape is anisotropic: the volume and surface integrals are difficult to express in suitable coordinates, so they are analytically intractable. No such calculation is presented in their paper. More recently, Rysakov et al. [5-7] suggested a new approximate method allowing the determination of the scattering properties of optically soft particles whatever their shape and size. His work 
is also based on the WKB approximation, and the scattering analysis is performed in the three-dimensional spectral space. Rysakov illustrated his method with simple shapes. However, its application to particle shape without symmetry element seems very difficult.

The aim of this article is to calculate the phase function of an optically soft particle whatever its convex shape. The content of the article is the following. The second section introduces the principles of the WKB approximation for the calculation of the optical properties of particles. Section three presents the new calculation of the phase function. Section four is devoted to the validation of the method and its application to some specific cases.

\section{WKB approximation for optical properties}

The WKB technique [8] is mainly used for the solution of Schrödinger's equation in the short wavelength limit. Saxon [9] applied the WKB approximation for the scattering of electromagnetic waves: the wave equation is written as an integral equation using a Green's function. This one allows for an approximation that has a physical sense. This equation yields the WKB result as its zeroth approximation. It should be underlined that Saxon's method can be applied to non-homogeneous materials with no restriction with regard to size and shape of particle. Ueberall[10] successfully used WKB method to determine the scattering of conductors coated with weakly scattering material. Deirmendjian [11] introduced this method for the calculation of the light scattering by spheres and proved its interest. Klett and Sutherland [4] extended the application of WKB to cylinders.

Following Klett and Sutherland, the WKB approximation considers:

- a rectilinear propagation of the incident wave in the scatterer (as in the RDG approximation)

- a change of phase of the electromagnetic wave, in proportion to the degree of penetration into the scatterer.

Thus the WKB approximation appears as a modification of the RDG approximation for which the amplitude form factor is given by:

$$
R=\frac{1}{V} \int e^{i \overrightarrow{k_{s}} \cdot \vec{r}} d V
$$

whereas the corresponding one for WKB is given by [4,9]: 
$R=\frac{1}{V} \int e^{i\left[\vec{k}_{s} \cdot \vec{r}+(m-1) \vec{k} \cdot(\vec{r}-\vec{r})\right]} d V$

Integration is performed over the body volume. $\vec{k}, \theta$ and $-\vec{k}_{s}\left(k_{s}=2 k \sin (\theta / 2)\right)$ are the incident wave vector, the scattering angle and the scattering vector, respectively. The vector $\vec{k}_{s}$ has been defined following Klett [4]. V, $\vec{r}$ and $\overrightarrow{r^{\prime}}$ are the object volume, the position of the scattering element inside the object and the initial position of penetration of the object. The expression $1 \mathrm{~b}$ is close to the one given by Van de Hulst ([2], p. 184) but the latter is established for a sphere and for very small scattering angles. It may be noted that the additional term in Eq.(1b) breaks the symmetry of Eq.(1a).

The differential scattering cross section per unit incident intensity (unpolarized light) is related to the amplitude form factor as:

$C_{\text {diff sca }}=\frac{k^{4} V^{2}}{4 \pi^{2}}|m-1|^{2}|R|^{2} \frac{1+\cos ^{2} \theta}{2}$

The following section presents an original contribution to the calculation of the amplitude form factor.

\section{Calculation of the amplitude form factor}

Let us consider a non absorbing object illuminated by an unpolarized plane wave. The object has a fixed orientation. The penetration of the light ray into the body is explicitly considered in the WKB approximation. As a consequence, the propagation direction is a preferential line that strengthens the anisotropy of the physical phenomenon and its solving. One integrates Eq.(1b) along the line parallel to the $\vec{k}$ vector, i.e. z-axis (figure 1). The initial penetration position has a fixed value during the z-integration. This leads to the following expression for the amplitude form factor:

$R=\frac{1}{i b V} \iint\left[e^{i\left(k_{s}[x u+y v]-(m-1) k z^{+}\right)}\left(e^{i b z^{-}}-e^{i b z^{+}}\right)\right] d x d y$

with

$b=k_{s} w+(m-1) k$ 
$\mathrm{x}, \mathrm{y}$ and $\mathrm{z}$ are the components of $\vec{r} . \mathrm{u}, \mathrm{v}$ and $\mathrm{w}$ are the components of the unit vector $\overrightarrow{k_{s}} / k_{s}$. The coordinate system is composed of $\vec{k} / k$ and two unit vectors in the plane perpendicular to $\vec{k}$ : the components of $\overrightarrow{k_{s}}$ are $k(-\sin \theta \cos \varphi,-\sin \theta \sin \varphi, 1-\cos \theta)$ with $\varphi$ the azimuthal angle. The domain of integration is the projection of the body on the plane perpendicular to the $\vec{k}$ vector. $z^{+}$and $z^{-}$are the $\mathrm{z}$-coordinates of the intersection of a line parallel to $\vec{k}$ and the body surface (figure 1).

Then one integrates over the $y$ variable. For this purpose, the intersection of the object and the $(\mathrm{yOz})$ plane (figure 2) at $x=x_{p}$ is considered. We call $(\mathrm{C})$ the closed curve that bounds the intercept. The centre of mass of the interior of $(\mathrm{C})$ is noted $\mathrm{O}$. Its y-coordinate and its z-coordinate are noted respectively by $y^{0}$ and $z^{0}$. The closed curve and its characteristics depend on the x-coordinate. As a consequence, Eq. (3) can be written as:

$$
R=\frac{1}{V} \int F_{p} d x_{p}
$$

with

$$
F_{p}=F\left(x_{p}\right)=\frac{1}{i b} e^{i\left(x_{p} u+y_{p}^{0} v+z_{p}^{0} w\right) k_{s}}\left(G_{p}^{-}-G_{p}^{+}\right)=F_{p}^{r}+i F_{p}^{i}
$$

and

$$
G_{p}^{ \pm}=\int_{y_{p, \text { min }}}^{y_{p, \max }} e^{-i(m-1) k\left(z_{p}^{+}-z_{p}^{0}\right)+i\left((m-1) k+k_{s} w\right)\left(z_{p}^{ \pm}-z_{p}^{0}\right)+i k_{s} v\left(y_{p}-y_{p}^{0}\right)} d y_{p}
$$

with $y_{p}^{0}=y^{0}\left(x_{p}\right) \quad z_{p}^{0}=z^{0}\left(x_{p}\right) \quad z_{p}^{ \pm}=z^{ \pm}\left(x_{p}, y_{p}\right)$

The equation (5) leads to:

$$
|R|^{2}=\frac{1}{V^{2}}\left[\left(\int_{x \min }^{x \max } F^{r}\left(x_{p}\right) d x_{p}\right)^{2}+\left(\int_{x \min }^{x \max } F^{i}\left(x_{p}\right) d x_{p}\right)^{2}\right]
$$

To have an analytical estimate of $G_{p}^{ \pm}$, the intercept $(\mathrm{C})$ is replaced by an ellipse (E) (figure 2). The latter is characterized by its semi-major axis $A$, its semi-minor axis $B$ and the angle $\alpha(0 \leq \alpha \leq \pi)$ between the semi-axis $A$ and z-axis. The choice of $A, B$ and $\alpha$ will be discussed in the paragraph 4.5. $A, B$ and $\alpha$ are functions of $x$. For the $\left(\mathrm{E}_{\mathrm{p}}\right)$ ellipse, Eq.(9) can be derived:

$$
z_{p}^{ \pm}-z_{p}^{0}=\left[ \pm A_{p} B_{p}\left(h_{0, p}^{2}-\left(y_{p}-y_{p}^{0}\right)^{2}\right)^{1 / 2}+\left(y_{p}-y_{p}^{0}\right)\left(A_{p}^{2}-B_{p}^{2}\right) \sin \alpha_{p} \cos \alpha_{p}\right] / h_{0, p}^{2}
$$

with 
$h_{0, p}=h_{0}\left(x_{p}\right)=\left(B_{p}^{2} \cos ^{2} \alpha_{p}+A_{p}^{2} \sin ^{2} \alpha_{p}\right)^{1 / 2}$

As $y_{p, \text { max }}-y_{p}^{0}=-y_{p, \min }+y_{p}^{0}=h_{0, p}$ we can write:

$y_{p}-y_{p}^{0}=h_{0, p} \sin \beta$ and $-\pi / 2 \leq \beta \leq \pi / 2$

Then

$z_{p}^{ \pm}-z_{p}^{0}=h_{1, p} \sin \left(\beta \pm \gamma_{p}\right)$

with

$h_{1, p}=\left(B_{p}^{2} \sin ^{2} \alpha_{p}+A_{p}^{2} \cos ^{2} \alpha_{p}\right)^{1 / 2}$

and

$$
\gamma_{p}=\arcsin \left(A_{p} B_{p} /\left(h_{0, p} h_{1, p}\right)\right) \quad \text { with } \operatorname{sign}\left(\gamma_{p}-\pi / 2\right)=\operatorname{sign}\left(\alpha_{p}-\pi / 2\right)
$$

By introducing Eq.(11) in Eq.(7) (see Appendix for details):

$G_{p}^{ \pm}=h_{0, p} \int_{-\pi / 2}^{\pi / 2} e^{i q_{p}^{ \pm} \sin \left(\beta+t_{p}^{ \pm}\right)+i q_{p}^{0} \sin \beta} \cos \beta d \beta=h_{0, p} \int_{-\pi / 2}^{\pi / 2} e^{i r_{p}^{ \pm} \sin \left(\beta+\delta_{p}^{ \pm}\right)} \cos \beta d \beta$

With

$$
\begin{aligned}
& H_{0}=k_{s} v, H_{+}=k_{s} w, H_{-}=k_{s} w+2(m-1) k \\
& q_{p}^{0}=H_{0} h_{0, p} \\
& q_{p}^{+}=H_{+} h_{1, p} \\
& t_{p}^{+}=\gamma_{p} \\
& q_{p}^{-}=h_{1, p}\left(H_{+}^{2} \cos ^{2} \gamma_{p}+H_{-}^{2} \sin ^{2} \gamma_{p}\right)^{1 / 2} \\
& t_{p}^{-}=-\arcsin \left(H_{-} h_{1, p} \sin \gamma_{p} / q_{p}^{-}\right) \quad \text { with } \operatorname{sign}\left(t_{p}^{-}+\pi / 2\right)=\operatorname{sign}\left(\alpha_{p}-\pi / 2\right) \\
& r_{p}^{ \pm}=\left(q_{p}^{ \pm 2}+q_{p}^{02}+2 q_{p}^{ \pm} q_{p}^{0} \cos t_{p}^{ \pm}\right)^{1 / 2} \\
& \delta_{p}^{ \pm}=\arcsin \left(q_{p}^{ \pm} \sin t_{p}^{ \pm} / r_{p}^{ \pm}\right) \quad \text { with } \quad \operatorname{sign}\left(\pi / 2 \mp \delta_{p}^{ \pm}\right)=\operatorname{sign}\left(q_{p}^{ \pm} \cos t_{p}^{ \pm}+q_{p}^{0}\right)
\end{aligned}
$$

Taking into account the properties of the Bessel functions $J_{v}$ of integer order [12], Eq.(16) is obtained (see Appendix for details):

$$
G_{p}^{ \pm}=2 h_{0, p}\left[J_{0}\left(r_{p}^{ \pm}\right)-2 \sum_{n=1}^{\infty} \frac{(-1)^{n}}{\left(4 n^{2}-1\right)} J_{2 n}\left(r_{p}^{ \pm}\right) \cos \left(2 n \delta_{p}^{ \pm}\right)+i \frac{\pi}{2} J_{1}\left(r_{p}^{ \pm}\right) \sin \left(\delta_{p}^{ \pm}\right)\right]
$$

Thereafter, $G_{p}^{+}$and $G_{p}^{-}$are introduced in Eq.(6) to calculate $F_{p}^{r}$ and $F_{p}^{i}$, themselves used in Eq.(8) to calculate $|R|^{2}$. The series in (16) is convergent. A small number $(\leq 2)$ of series terms is sufficient to get accurate values of $|R|^{2}$. 
Let us consider the RDG approximation that corresponds to a zero phase shift or $|m-1| \rightarrow 0$. For this particular case, Eqs.(15) and (16) lead to a reduced expression: $G_{p}^{-}-G_{p}^{+}=i 2 \pi h_{0, p} J_{1}\left(r_{p}^{+}\right) \sin \delta_{p}^{+}$

It will be underlined that the real part in (16) does not contribute in the framework of the RDG approximation.

As a consequence,

$$
F_{p}=e^{i\left(x_{p} u+y_{p}^{0} v+z_{p}^{0} w\right) k_{s}} 2 S_{p} \frac{J_{1}\left(r_{p}^{+}\right)}{r_{p}^{+}}
$$

$S_{p}$ is the area of the ellipse. Eqs. (8) and (17) lead to the expression of $|R|$.

\section{Applications}

If all the geometrical cross sections are ellipses in the object, e.g. for ellipsoidal particles, the result obtained with Eqs. (6), (8) and (16) is exact.

Some particular cases will now be examined.

\subsection{The cross section is chosen as a circle}

Circle is a generic shape for the geometrical cross section. It is a peculiar case of an ellipse:

$$
\begin{aligned}
& A=B=h_{0}=h_{1}, \\
& q_{p}^{0}=H_{0} h_{0, p} \\
& q_{p}^{+}=H_{+} h_{0, p} \\
& t_{p}^{+}=\pi / 2 \\
& q_{p}^{-}=H_{-} h_{0, p} \\
& t_{p}^{-}=-\pi / 2 \\
& r_{p}^{ \pm}=\left(q_{p}^{ \pm 2}+q_{p}^{02}\right)^{1 / 2} \\
& \delta_{p}^{ \pm}=\arcsin \left( \pm q_{p}^{ \pm} / r_{p}^{ \pm}\right)
\end{aligned}
$$

\subsection{The object is a sphere}


If the object is a sphere with radius $r$ and the incident light is unpolarized, the phase function does not depend on the $\varphi$ angle (this is equivalent to $\varphi=0$ ). The additional relations are to be considered:

$h_{0}=\left(r^{2}-x^{2}\right)^{1 / 2}, x_{\min }=-r, x_{\max }=r, y^{0}=0, z^{0}=0$

Thus $|R|^{2}$ only needs to estimate the integrals:

$I_{p}=\frac{1}{b} \int_{-r}^{+r} \cos \left(a_{T} x\right)\left(r^{2}-x^{2}\right)^{1 / 2} J_{p}\left(a_{J \pm}\left(r^{2}-x^{2}\right)^{1 / 2}\right) d x$

with $p=1$ or $p=2 n, a_{T}=k_{s} u, a_{J \pm}=H_{ \pm}$.

$I_{p}$ is a convolution product. Hardy proposed the following expression for a related integral (in [13] p47):

$$
\begin{aligned}
& K_{p \pm}=\int_{0}^{t} \tau^{-1 / 2} J_{p}\left(a_{J \pm} \tau^{1 / 2}\right)(t-\tau)^{-1 / 2} \cos \left(a_{T}(t-\tau)^{1 / 2}\right) d \tau \\
& =\pi J_{p / 2}\left(\frac{1}{2} t^{1 / 2}\left(\left(a_{J \pm}^{2}+a_{T}^{2}\right)^{1 / 2}+a_{T}\right)\right) J_{p / 2}\left(\frac{1}{2} t^{1 / 2}\left(\left(a_{J \pm}^{2}+a_{T}^{2}\right)^{1 / 2}-a_{T}\right)\right)
\end{aligned}
$$

It is easy to show that $I_{p}$ and $K_{p}$ are related by the equation (20):

$$
I_{p}=\frac{1}{b}\left(\frac{\partial^{2} K_{p}}{\partial a_{T}^{2}}+r^{2} K_{p}\right)
$$

Thus,

$$
\begin{aligned}
& |R|^{2} V^{2}=\left[\frac{2}{b}\left\{\frac{\partial^{2} K_{0}}{\partial a_{T}^{2}}+r^{2} K_{0}-2 \sum_{n=1}^{\infty} \frac{1}{\left(4 n^{2}-1\right)}\left(\frac{\partial^{2} K_{2 n}}{\partial a_{T}^{2}}+r^{2} K_{2 n}\right)\right\}_{a_{J+}}^{a_{J-}}\right]^{2} \\
& +\left[\frac{2}{b}\left\{ \pm \frac{\pi}{2}\left(\frac{\partial^{2} K_{1}}{\partial a_{T}^{2}}+r^{2} K_{1}\right)\right\}_{a_{J+}}^{a_{J-}}\right]^{2}
\end{aligned}
$$

$|R|$ is only expressed by means of the Bessel functions.

If $a_{J_{+}}=a_{J_{-}}$, i.e. within the validity range of RDG approximation, only $I_{1}$ contributes to $|R|$ :

$$
|R|=\frac{2 \pi}{b V}\left(\frac{\partial^{2} K_{1}}{\partial a_{T}^{2}}+r^{2} K_{1}\right)=3\left(\left(k_{s} r\right) \cos \left(k_{s} r\right)-\sin \left(k_{s} r\right)\right) /\left(k_{s} r\right)^{3}
$$

which is the well-known result of the RDG approximation for a sphere [2].

The figures $3 \mathrm{a}-\mathrm{b}$ compare $|R|^{2}$ versus the scattering angle calculated from Mie theory, RDG and $\mathrm{AD}$ approximations and issued from this work. Figure $3 \mathrm{a}$ corresponds to a sphere for which the scattering cross section does not obey the AD approximation: as 
expected, the values calculated in this work are very close to the ones obtained from Mie theory and RDG approximation. Figure $3 \mathrm{~b}$ corresponds to a sphere for which the scattering cross section slightly obeys the $\mathrm{AD}$ approximation: as expected, RDG approximation does not work. There is a good agreement between Mie calculation and this work particularly at small angle, i.e. at high scattered intensity value; it can be underlined that, contrary to data coming from $\mathrm{AD}$ approximation, the location of the secondary maxima calculated in this work is in agreement with Mie theory.

\subsection{The object is a spheroid}

A spheroid with semi-axis ( $a s, a s, b s)$ is considered. The angle between $b s$ axis and the incident wave vector is noted $\Psi$. Then,

$$
\begin{aligned}
& r=\left(b s^{2} \sin ^{2} \Psi+a s^{2} \cos ^{2} \Psi\right)^{1 / 2}, \quad h_{0}=\frac{a s}{r}\left(r^{2}-x^{2}\right)^{1 / 2}, \quad x_{\min }=-r, \quad x_{\max }=r, y^{0}=0 \\
& z^{0}=\frac{b s^{2}-a s^{2}}{r^{2}} \sin \psi \cos \psi x \\
& a_{T} \text { and } a_{J \pm} \text { are now: } \\
& a_{T}=k_{s} u+H_{+} \frac{b s^{2}-a s^{2}}{r^{2}} \sin \Psi \cos \Psi \\
& a_{J \pm}=\left[\left(H_{ \pm} \frac{a s b s}{r^{2}}\right)^{2}+\left(H_{0} \frac{a s}{r}\right)^{2}\right]^{1 / 2}
\end{aligned}
$$

The form factor is a modified version of the equation (21):

$$
\begin{aligned}
& |R|^{2} V^{2}=\left(\frac{a s}{r}\right)^{2}\left[\frac{2}{b}\left\{\frac{\partial^{2} K_{0}}{\partial a_{T}^{2}}+r^{2} K_{0}-2 \sum_{n=1}^{\infty} \frac{(-1)^{n}}{\left(4 n^{2}-1\right)}\left(\frac{\partial^{2} K_{2 n}}{\partial a_{T}^{2}}+r^{2} K_{2 n}\right) \cos (2 n \delta)\right\}_{a_{J+}}^{a_{J-}}\right]^{2} \\
& +\left(\frac{a s}{r}\right)^{2}\left[\frac{2}{b}\left\{\frac{\pi}{2}\left(\frac{\partial^{2} K_{1}}{\partial a_{T}^{2}}+r^{2} K_{1}\right) \sin \delta\right\}_{a_{J+}}^{a_{J-}}\right]^{2}
\end{aligned}
$$

Numerous investigators studied the scattering properties of spheroids. Latimer [14] and Latimer and Barber [15] proposed improved methods to calculate the differential scattering cross sections of ellipsoids of revolution. They are firstly based on the determination of radius and refractive index of their equivalent sphere. Then, the scattered intensity of the sphere is calculated from the Mie theory. Finally the scattering intensity of the ellipsoid is obtained by multiplying the scattering intensity of the sphere by a correction factor. One of these two methods denoted RM-I by Latimer is suitable 
for small size parameter. The other (denoted AM-II by Latimer) fits well for large size parameter. Chen [16] and Chen and Yang [17] have obtained the same results (AM-II) for spheroids with a large size parameter. Rysakov [5-7] has calculated the optical properties of spheroids. Two points must be underlined:

- for a spheroid with a symmetry axis having a direction different from the propagation direction, the differential scattering cross section is not an even function of the scattering angle

- there is a small angular shift of the principal maxima. Larger the relative refractive index is, larger the shift value is.

A comparison of these different methods applied to a spheroid shows that:

- under RDG approximation conditions, $|R|=G_{D}(x)$ with $G_{D}(x)=3(x \cos x-\sin x) / x^{3}$ for Rysakov whereas $|R| \stackrel{m \rightarrow 1}{\longrightarrow} G_{D}(x)$ for Latimer.

In these two works, $x$ has the same meaning: $x=k_{s} a s\left(\sin ^{2} \eta+(b s / a s)^{2} \cos ^{2} \eta\right)^{1 / 2} \cdot \eta$ is the angle between the scattering vector and the symmetry axis of the spheroid.

- under $\mathrm{AD}$ approximation conditions, there is a significant difference between the results of Rysakov, Latimer and Chen. However, Rysakov's calculations are closer to the AM-II method than to the RM-I method at small angle.

Figures 4a-b show $|R|^{2}(\theta)$ calculated following RM-I, AM-II and Rysakov methods, and following this work:

- under RDG approximation conditions (figure 4a), a good agreement between the four methods is observed.

- under AD approximation conditions (figure 4b), for which $m-1$ is not so small, AM-II and our method are in good agreement. As expected, RM-I is not suitable whereas the Rysakov's curve is similar to our data, but with an angular shift.

\subsection{The object is a cylinder}

If the object is a finite cylinder with length $2 L$ and if the light is normally incident upon the cylinder, then: $x_{\min }=-L, x_{\max }=L, y^{0}=0, z^{0}=0 . G^{ \pm}$does not depend on $x$. Thus, $|R|$ obeys the expression:

$$
|R|=\frac{2}{b V} \frac{\sin k_{s} u L}{k_{s} u}\left|G^{-}-G^{+}\right|
$$


Eq. 24 has been successfully compared with Eqs. $43 \mathrm{a}$ and $43 \mathrm{~b}$ (for $h_{0}=h_{1}=r$ ) in the work of Klett [4].

\subsection{Any object}

For any shapely scatterer the parameters $\left(y^{0}, z^{0}, A, B\right.$ and $\left.\alpha\right)$ of the equivalent ellipse for each geometrical cross section ( $x=$ constant) are needed. A simple way to perform it consists in the following steps [18]:

- Deduce from the surface equation of the object as $f(x, y, z)=0$ the closed (C) curve equation corresponding to $x$-constant,

- Calculate the normalized moments $M_{i j}(x)=\int_{[C]} y^{i} z^{j} d y d z / \int_{[C]} d y d z$ with $i+j \leq 2$ (i and $\mathrm{j}$ are non negative integers); $[\mathrm{C}]$ is the interior of $\mathrm{C}$,

- Deduce the centered moments $m_{i j}(x)$ with $i+j=2$,

- Calculate the eigenvalues $\lambda_{1}, \lambda_{2}\left(\lambda_{1}>\lambda_{2}\right)$ of the matrix $\left[\begin{array}{ll}m_{20} & m_{11} \\ m_{11} & m_{02}\end{array}\right]$

$A, B$ and $\alpha$ are given by:

$$
A=2 \lambda_{1}^{1 / 2}, B=2 \lambda_{2}^{1 / 2}, \alpha=\frac{1}{2} \arctan \left(\frac{2 m_{11}}{m_{20}-m_{02}}\right)
$$

In order to illustrate this procedure, one considers the scattering of light by a cylinder with the symmetry axis aligned on the propagation direction. Three methods are used for calculating $|R|^{2}(\theta)$ : Klett [4], Rysakov [7] and our method. These object shape and orientation have been selected according to the nature of the intercept of the object by a $x=$ cte plane: the intercept is a rectangle with side lengths $2 L$ and $2\left(r^{2}-x^{2}\right)^{1 / 2}$ which is very different from a rounded curve like an ellipse. The equivalent ellipse is characterized by:

$$
\begin{aligned}
& A=2 L / \sqrt{3} \\
& B=2\left(r^{2}-x^{2}\right)^{1 / 2} / \sqrt{3} \\
& \alpha=0 \\
& y_{0}=0 \\
& z_{0}=0
\end{aligned}
$$

The figure 5 compares the data corresponding to the three methods. The agreement is good for the entire $\theta$ angular range. However, it may be not quite as good for other 
azimuthal angles. Even in this case the agreement is very good for the small $\theta$ angle range, i.e. corresponding to $|R|^{2}(\theta)>10^{-3}$. For a cylinder with such orientation, the described procedure leads to a loss of the symmetry around z-axis which implies small changes of $|R|^{2}(\theta)$ when the azimuthal $\varphi$ angle is varied.

\section{Conclusion}

The presented calculation of the amplitude form factor for light scattering at soft scatterers, i.e. $|m-1|<<1$, proceeds by two steps:

- a geometrical one requiring the determination of the parameters of the equivalent ellipse for any cross section. If the cross section is rigorously an ellipse, this first step is skipped.

- an optical one calculating exactly the amplitude form factor thanks to equations 6,8 and 16 which are derived from the principles of RDG and AD approximations

Its implementation into a high level computer language can be very short and such a code is much easier to review than a T-matrix code for instance. The calculation is the more accurate, the closer the geometrical cross sections resemble an ellipse. At the opposite if the cross sections are parallelograms, the calculated value of the form factor can be expected to be less accurate. So it is suggested to modify the generic shape of the cross section (an ellipse in this paper) for studying polyhedrons as crystals. This work is in progress.

\section{References}

[1] F.M. Kahnert, Numerical methods in electromagnetic scattering theory, J. Quant. Spectr. Rad. Transfer 79 (2003) 775-824.

[2] H.C. Van de Hulst, Light scattering by small particles, J.Wiley, New York, 1957.

[3] A.Y. Perelman, Extinction and scattering by soft spheres, Applied Optics 30 (1991) 475-484.

[4] J.D. Klett, R.A. Sutherland, Approximate methods for modelling the scattering properties of nonspherical particles: evaluation of the Wentzel-Kramers-Brillouin method, Applied Optics 31 (1992) 373-386.

[5] W. Rysakov, M. Ston', Light scattering by spheroids, J. Quantitative Spectroscopy \& Radiative Transfer 69(2001)651-665. 
[6] W. Rysakov, Light scattering by soft particles of arbitrary shape and size, J. Quantitative Spectroscopy \& Radiative Transfer 87(2004)261-287.

[7] W. Rysakov, Light scattering by soft particles of arbitrary shape and size: IIArbitrary orientation of particles in the space, J. Quantitative Spectroscopy \& Radiative Transfer 98(2006)85-100.

[8] G. Wentzel, Eine Verallgemeinerung der Quantumbedingungen fuer die Zwecke der Wellenmechanik, Zeitschrift fuer Physik 38 (1926) 518-529.

[9] D.S. Saxon, Modified WKB methods for the propagation and scattering of electromagnetic waves, IRE transactions on antennas and propagation (1959) S320-323. [10] H. Ueberall, Radar scattering from coated perfect conductors: application to the semi-infinite cone and use of exact eikonal, Radio Science Journal of Research NBS/USNC-URSI (1964) 68D:6.

[11] D. Deirmendjian, Theory of the solar aureole, part II: applications to atmospheric models, Ann. Geophys. 15 (1959) 218-249.

[12] M. Abramowitz, I.A. Stegun, Handbook of mathematical functions, Dover publications, New York, 1972.

[13] A. Erdélyi, Higher transcendental functions, Vol II, Mac Graw Hill book company, New York, 1953.

[14] P. Latimer, Light scattering by ellipsoids, J. Coll. And Interf. Science 53(1975)102-109.

[15] P. Latimer, P Barber, Scattering by ellipsoids of revolution A comparison of theoretical methods, J. Coll. And Interf. Science 63(1978)310-316.

[16] T.W. Chen, Effective sphere for spheroid in light scattering, Optics Communications 114(1995)199-202.

[17] T.W. Chen, L.M. Yang, Simple formula for small-angle light scattering by a spheroid, Optics Communications 123(1996)437-442.

[18] R. Safaee-Rad, K.C. Smith, B. Benhabib, I. Tchoukanov, Application of moment and Fourier descriptors to the accurate estimation of elliptical-shape parameters, Pattern Recognition Letters 13(1992)497-508. 


\section{Appendix}

Let us consider the Eq.7

$G_{p}^{ \pm}=\int_{y_{p, \text { min }}}^{y_{p, \text { max }}} e^{-i(m-1) k\left(z_{p}^{+}-z_{p}^{0}\right)+i\left((m-1) k+k_{s} w\right)\left(z_{p}^{ \pm}-z_{p}^{0}\right)+i k_{s} v\left(y_{p}-y_{p}^{0}\right)} d y_{p}$

and

$$
\begin{aligned}
& y_{p, \max }-y_{p}^{0}=-y_{p, \min }+y_{p}^{0}=h_{0, p} \\
& y_{p}-y_{p}^{0}=h_{0, p} \sin \beta \\
& z_{p}^{ \pm}-z_{p}^{0}=h_{1, p} \sin \left(\beta \pm \gamma_{p}\right)
\end{aligned}
$$

Let us now examine the case $z^{ \pm}=z^{+}$. The case $z^{ \pm}=z^{-}$may be similarly treated.

By introducing Eq.(11) in Eq.(7):

$$
G_{p}^{+}=h_{0, p} \int_{-\pi / 2}^{\pi / 2} e^{i k_{s} w h_{1, p} \sin \left(\beta+\gamma_{p}\right)+i k_{s} v h_{0, p} \sin \beta} \cos \beta d \beta
$$

By denoting

$H_{0}=k_{s} v, H_{+}=k_{s} w$

$q_{p}^{0}=H_{0} h_{0, p}$

$q_{p}^{+}=H_{+} h_{1, p}$

$t_{p}^{+}=\gamma_{p}$

$G_{p}^{+}=h_{0, p} \int_{-\pi / 2}^{\pi / 2} e^{i q_{p}^{+} \sin \left(\beta+t_{p}^{+}\right)+i q_{p}^{0} \sin \beta} \cos \beta d \beta$

or

$G_{p}^{+}=h_{0, p} \int_{-\pi / 2}^{\pi / 2} e^{i\left(q_{p}^{+} \cos \left(t_{p}^{+}\right)+q_{p}^{0}\right) \sin \beta+i q_{p}^{+} \sin \left(t_{p}^{+}\right) \cos \beta} \cos \beta d \beta$

By denoting

$$
\begin{aligned}
& r_{p}^{+}=\left(q_{p}^{+2}+q_{p}^{02}+2 q_{p}^{+} q_{p}^{0} \cos t_{p}^{+}\right)^{1 / 2} \\
& \delta_{p}^{+}=\arcsin \left(q_{p}^{+} \sin t_{p}^{+} / r_{p}^{+}\right) \quad \text { with } \quad \operatorname{sign}\left(\pi / 2 \mp \delta_{p}^{+}\right)=\operatorname{sign}\left(q_{p}^{+} \cos t_{p}^{+}+q_{p}^{0}\right)
\end{aligned}
$$

Thus,

$$
G_{p}^{+}=h_{0, p} \int_{-\pi / 2}^{\pi / 2} e^{i r_{p}^{+} \sin \left(\beta+\delta_{p}^{+}\right)} \cos \beta d \beta
$$

For the sake of simplicity, let us consider the integral (without subscript and superscript for $r_{p}^{+}$and $\delta_{p}^{+}$):

$G=h_{0} \int_{-\pi / 2}^{\pi / 2} e^{i r \sin (\beta+\delta)} \cos \beta d \beta$ 
Then $G$ can be written (with $\alpha=\beta+\delta$ ):

$G=G_{1}+G_{2}$

with

$$
\begin{aligned}
& G_{1}=h_{0} \cos \delta \int_{-\pi / 2+\delta}^{\pi / 2+\delta} e^{i r \sin \alpha} \cos \alpha d \alpha=2 h_{0} \cos \delta \sin (r \cos \delta) / r \\
& G_{2}=h_{0} \sin \delta \int_{-\pi / 2+\delta}^{\pi / 2+\delta} e^{i r \sin \alpha} \sin \alpha d \alpha
\end{aligned}
$$

$G_{2}$ can be expressed as a series of Bessel functions [12].

as

$e^{i r \sin \alpha}=\cos (r \sin \alpha)+i \sin (r \sin \alpha)=J_{0}(r)+\sum_{n=1} 2 J_{2 n}(r) \cos (2 n \alpha)+i \sum_{n=0} 2 J_{2 n+1}(r) \cos ((2 n+1) \alpha)$ then

$e^{i r \sin \alpha} \sin \alpha=\sum_{n=0}\left(J_{2 n}(r)-J_{2 n+2}(r)\right) \sin ((2 n+1) \alpha)+i J_{1}(r)+i \sum_{n=1}\left(J_{2 n+1}(r)-J_{2 n-1}(r)\right) \cos (2 n \alpha)$

and

$$
G_{2}=h_{0} \sin \delta\left[\sum_{n=0} \frac{2(-1)^{n}}{2 n+1}\left(J_{2 n}(r)-J_{2 n+2}(r)\right) \sin ((2 n+1) \delta)+i \pi J_{1}(r)\right]
$$

Knowing that

$$
\sin (r \cos \delta)=\sum_{n=0} 2(-1)^{n} J_{2 n+1}(r) \cos ((2 n+1) \delta)
$$

and

$$
2 J_{2 n+1}(r)=r\left(J_{2 n}(r)+J_{2 n+2}(r)\right) /(2 n+1)
$$

then

$$
G=G_{1}+G_{2}=2 h_{0}\left[J_{0}(r)-2 \sum_{n=1}^{\infty} \frac{(-1)^{n}}{\left(4 n^{2}-1\right)} J_{2 n}(r) \cos (2 n \delta)+i \frac{\pi}{2} J_{1}(r) \sin (\delta)\right]
$$




\section{Captions}

Fig.1: Propagation of the electromagnetic waves into a scatterer in the case of WKB approximation.

Fig.2: Geometrical cross sections $(p=j, l)$ into a scatterer in the case of WKB approximation.

Fig. 3a: $|R|^{2}$ versus the scattering angle (radians) for a sphere $(m=1.05 ; \mathrm{kr}=2.5):+$ (cross): this work; dashed line: AD approximation; dash-dot line: RDG approximation; solid line: Mie theory.

Fig. 3b: $|R|^{2}$ versus the scattering angle (radians) for a sphere $(m=1.1 ; k r=25):+$ (cross): this work; dashed line: AD approximation; dash-dot line: RDG approximation; solid line: Mie theory.

Fig. 4a: $|R|^{2}$ versus the scattering angle (radians) for a prolate spheroid ( $m=1.01$; k.as $=2.5 ;$ bs/as $=5 ; \Psi=\pi / 3 ; \varphi=\pi / 4):+($ cross $):$ this work; dashed line: Rysakov's work; dash-dot line: AM-II; solid line: RM-I.

Fig. 4b: $|R|^{2}$ versus the scattering angle (radians) for a prolate spheroid ( $m=1.1$; k.as $=25 ;$ bs/as =5; $\Psi=\pi / 3 ; \varphi=\pi / 4)$ : + (cross): this work; dashed line: Rysakov's work; dash-dot line: AM-II; solid line: RM-I.

Fig. 5: $|R|^{2}$ versus the scattering angle (radians) for a cylinder $(m=1.01 ; k . r=5$; $L / r=0.5 ; \Psi=0 ; \varphi=0):+($ cross $):$ this work; dashed line: Rysakov's work; solid line: Klett's work. 


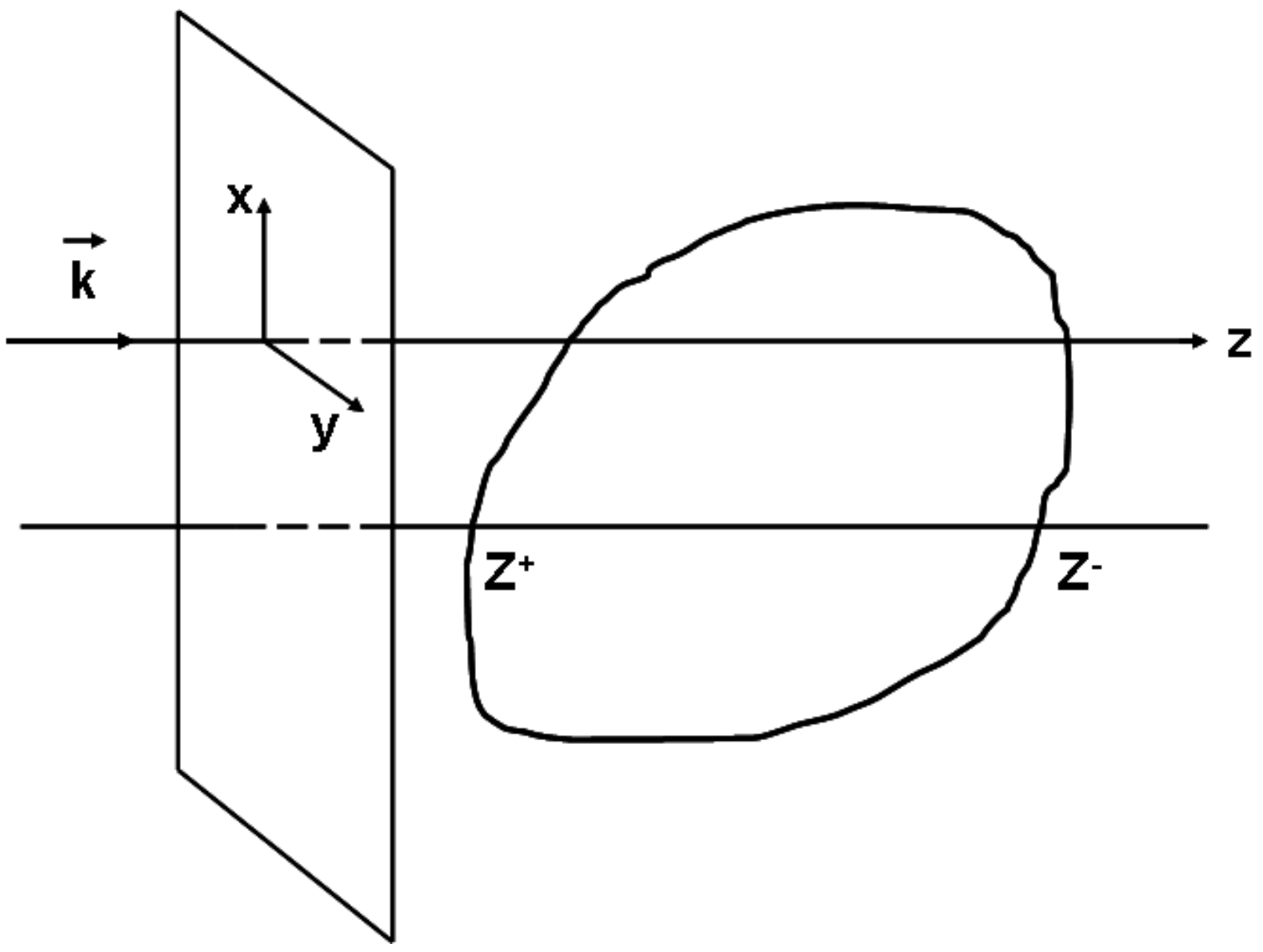

Figure 1 


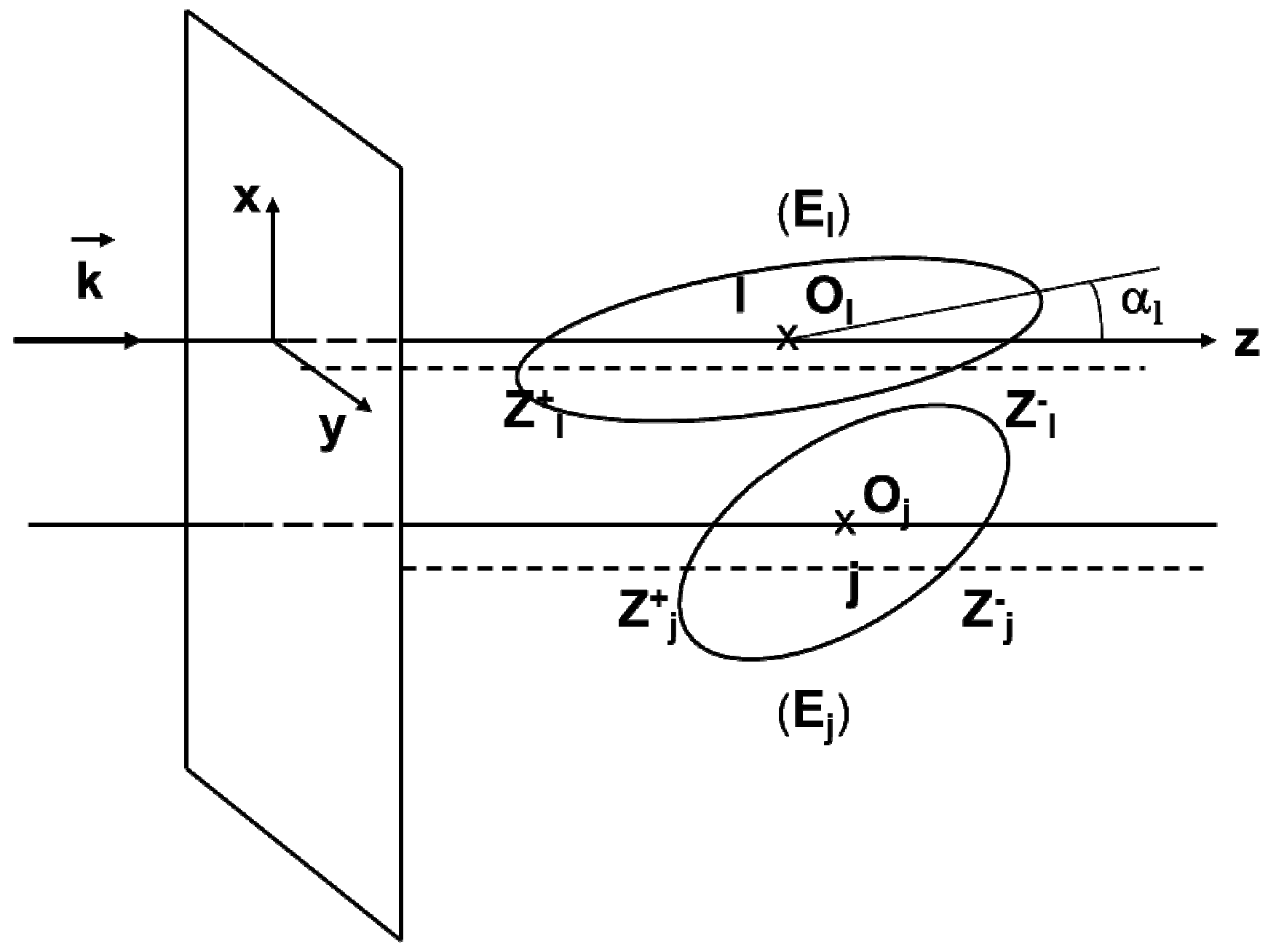

Figure 2 


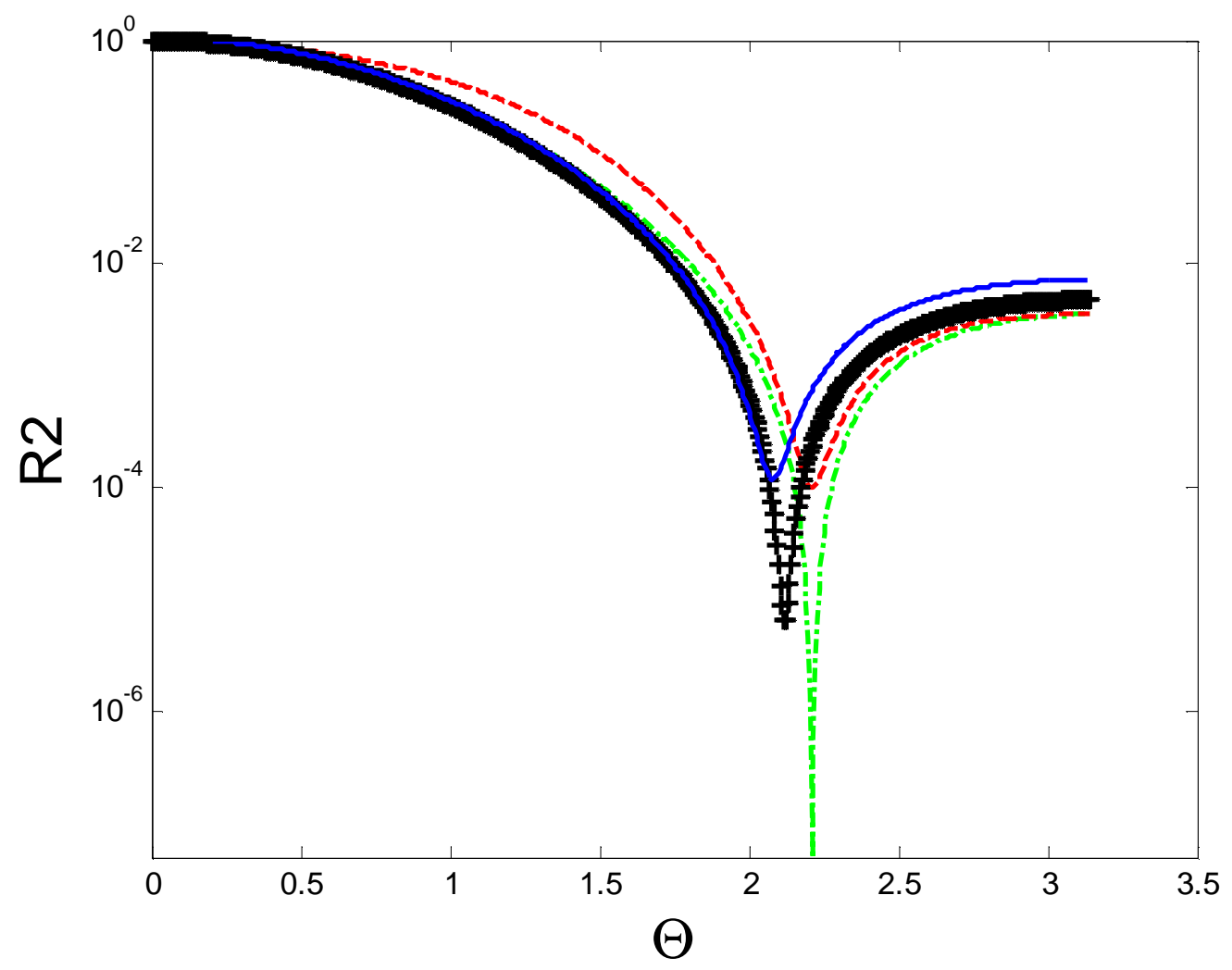

Figure 3a 


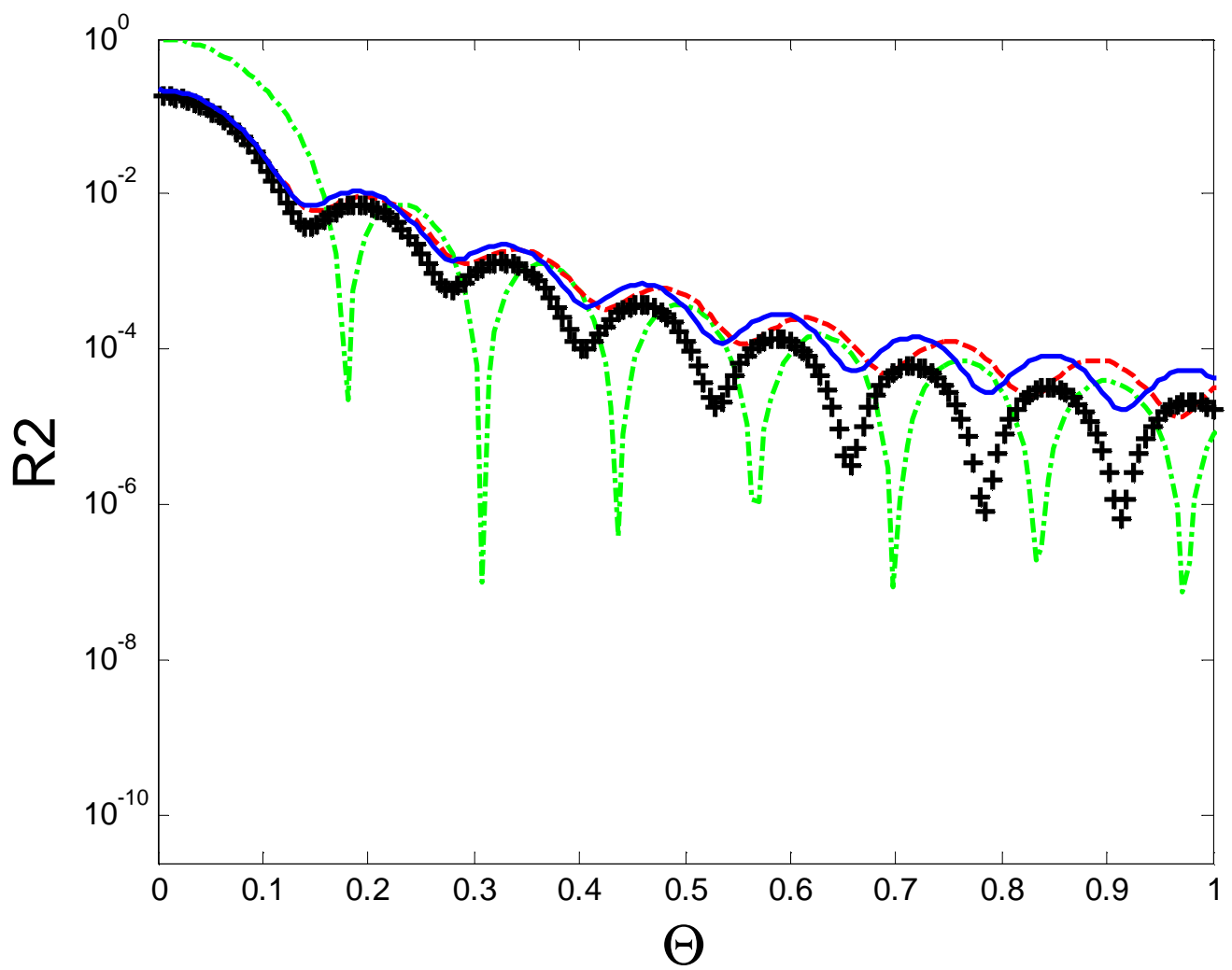

Figure 3b 


$$
\text { Int III }
$$




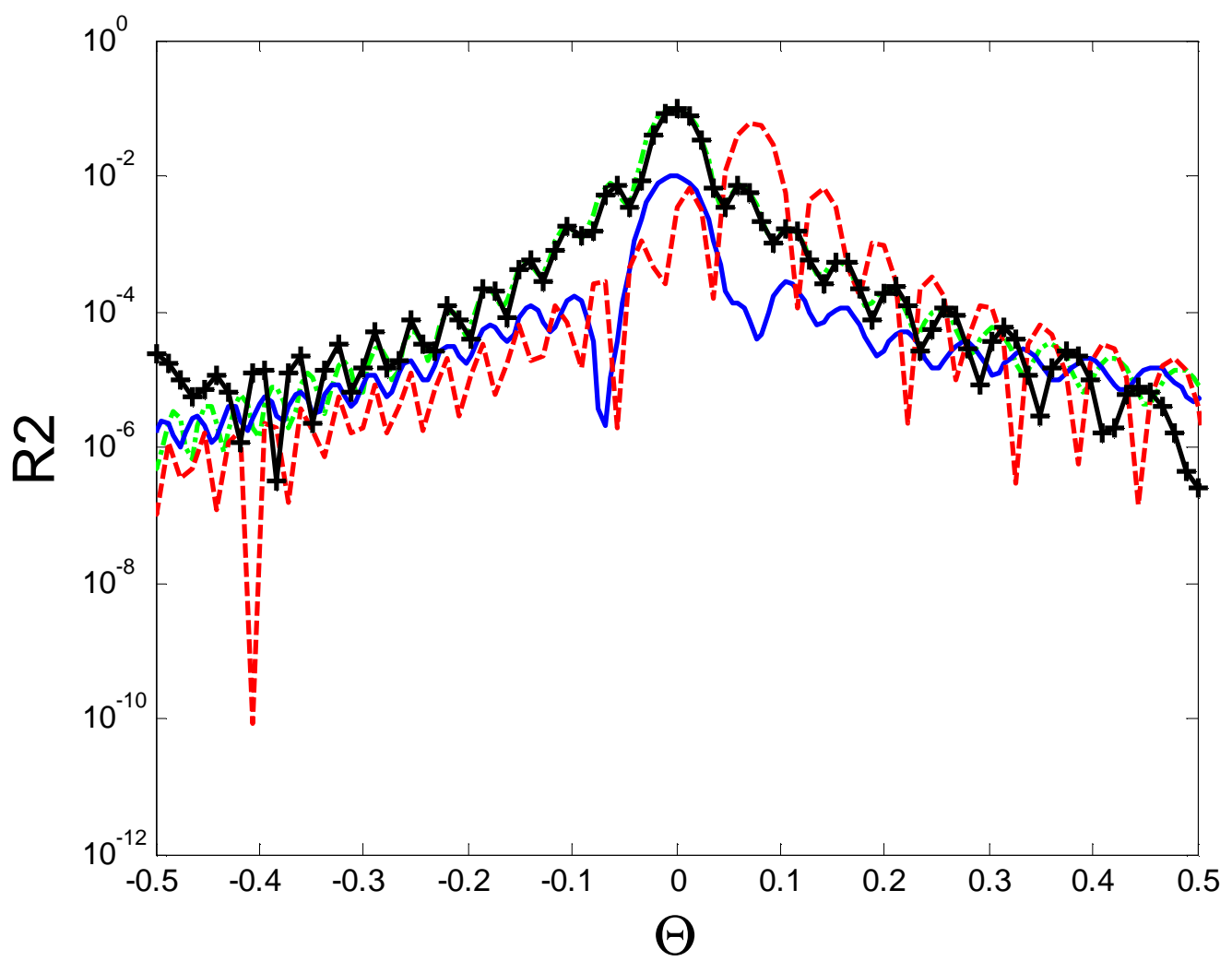

Figure 4b 


$$
\text { Br }
$$

